\title{
Clinicopathologic Significance of Survivin Expression in Relation to CD133 Expression in Surgically Resected Stage II or III Colorectal Cancer
}

\author{
Wanlu $\mathrm{Li}^{1} \cdot \mathrm{Mi}-\mathrm{Ra} \mathrm{Lee}^{1}$. \\ EunHee Choi ${ }^{2} \cdot$ Mee-Yon Cho ${ }^{1,3}$ \\ 'Department of Pathology, ${ }^{2}$ Institute of Lifestyle \\ Medicine, ${ }^{3}$ nstitute of Genomic Cohort, \\ Yonsei University Wonju College of Medicine, \\ Wonju, Korea
}

\author{
Received: July 18, 2016 \\ Revised: September 20, 2016 \\ Accepted: September 23, 2016 \\ Corresponding Author \\ Mee-Yon Cho, MD, PhD \\ Department of Pathology, Yonsei University Wonju \\ College of Medicine, 20 Ilsan-ro, Wonju 26426, \\ Korea \\ Tel: +82-33-731-1553 \\ Fax: +82-33-731-6590 \\ E-mail: meeyon@yonsei.ac.kr
}

\begin{abstract}
Background: Cancer stem cells have been investigated as new targets for colorectal cancer (CRC) treatment. We recently reported that $\mathrm{CD} 133^{+}$colon cancer cells showed chemoresistance to 5-fluorouracil through increased survivin expression and proposed the survivin inhibitor YM155 as an effective therapy for colon cancer in an in vitro study. Here, we investigate the relationship between survivin and CD133 expression in surgically resected CRC to identify whether the results obtained in our in vitro study are applicable to clinical samples. Methods: We performed immunohistochemical staining for survivin and CD133 in surgically resected tissue from 187 stage II or III CRC patients. We also comparatively analyzed apoptosis according to survivin and CD133 expression using terminal deoxynucleotidyl transferase-mediated deoxyuridine triphosphate nick-end labeling. Results: The results of the Mantel-Haenszel test established a linear association between nuclear survivin and CD133 expression $(p=.018)$, although neither had prognostic significance, according to immunohistochemical expression level. No correlation was found between survivin expression and the following pathological parameters: invasion depth, lymph node metastasis, or histologic differentiation ( $p>$.05). The mean apoptotic index in survivin ${ }^{+}$and CD133 tumors was higher than that in negative tumors: $5.116 \pm 4.894$ in survivin ${ }^{+}$versus $4.103 \pm 3.691$ in survivin- $(p=$ $.044) ; 5.165 \pm 4.961$ in $\mathrm{CD}_{133^{+}}$versus $4.231 \pm 3.812$ in $\mathrm{CD}^{2} 33^{-}(p=.034)$. Conclusions: As observed in our in vitro study, survivin expression is significantly related to CD133 expression. Survivin may be considered as a new therapeutic target for chemoresistant CRC.
\end{abstract}

Key Words: Colorectal neoplasms; Neoplastic stem cells; Survivin; CD133 protein; Apoptosis
Colorectal cancer (CRC) is the third most common cancer and the fourth most common cause of cancer-related death worldwide. ${ }^{1}$ Despite the availability of preventive measures, early screening tests, and improved surgical treatments and chemotherapy, the mortality rate remains high. The complexity of mechanisms involved in tumor recurrence, metastasis, and chemoresistance ${ }^{2}$ has spurred cancer stem cell studies to seek novel and more effective targets for CRC treatment. In our in vitro study, we found that $\mathrm{CD} 133^{+}$cells showed high levels of survivin expression, which was related to chemoresistance to 5-fluorouracil (5FU), and a survivin inhibitor was more effective than 5-FU in decreasing the viability of colon cancer cells. ${ }^{3,4}$

CD133, a transmembrane glycoprotein, is a cancer stem cell marker in several human tumors, ${ }^{5}$ including CRC. ${ }^{6}$ CD133 expression in $\mathrm{CRC}$ has been reported to be associated with $\mathrm{Wnt},{ }^{7,8}$ Notch, ${ }^{9}$ STAT $3,{ }^{10}$ and transforming growth factor-beta signaling pathways, ${ }^{11}$ which are essential for self-renewal, ${ }^{2}$ tumorigenesis, ${ }^{2}$ progression, ${ }^{12}$ and chemoresistance in CRC. ${ }^{3}$ However, the relationship of CD133 with survivin expression in clinical samples has not been evaluated.

Since tumor development and progression are based on the balance of cell apoptosis and proliferation, Alcaide et al. ${ }^{13}$ described that a higher rate of apoptosis is associated with more aggressive behavior of tumors and poorer prognosis in CRC patients. Survivin, a member of the inhibitor of apoptosis family, plays a dual role in cell apoptosis and proliferation. As a subunit of the chromosomal passenger complex, nuclear survivin regulates the chromosomal central spindle in various cell division processes, whereas cytoplasmic survivin inhibits apoptosis. This suggests that survivin can be a good target for cancer therapy because it promotes cell survival. ${ }^{14}$ In meta-analyses, survivin expression has shown a positive correlation with poorer prognosis in CRC patients. ${ }^{15,16}$ However, a previous study has reported that survivin expression was associated with good prognosis. ${ }^{17}$ Furthermore, most immunohistochemical studies of survivin did not clearly define the sublocation of survivin expression. Therefore, the clinical significance of survivin expression in CRC remains to be clarified.

The aim of the present study was to determine the clinico- 
pathologic significance of survivin expression in relation to CD133 expression in CRC. In addition, we comparatively analyzed apoptosis according to survivin and CD133 expression in CRC.

\section{MATERIALS AND METHODS}

\section{Patients and tissue samples}

We used formalin-fixed and paraffin-embedded tissues from surgically resected stage II or III CRCs that were registered to the Department of Pathology at Wonju Severance Christian Hospital between January 2000 and December 2006. We analyzed the data from 187 patients with follow-up information. Patients who received preoperative chemotherapy or radiotherapy were excluded. Clinicopathological data of patient age, sex, tumor location, invasion depth, histologic differentiation, and lymph node metastasis were collected from pathology reports.

\section{Ethics approval}

This study was approved by the Institutional Ethics Committee of Yonsei University, Wonju College of Medicine (YWMR14-4-102) and was carried out in compliance with the guidelines of the Declaration of Helsinki.

\section{Immunohistochemical staining}

Paraffin-embedded tissue sections from representative blocks were warmed to $75^{\circ} \mathrm{C}$ for 4 minutes. Slides were deparaffinized with EZPrep (Ventana Medical Systems, Tucson, AZ, USA), and an antigen-retrieval step was performed for 60 minutes with cell conditioning solution \#1 for survivin staining and \#2 for CD133 staining (Ventana Medical Systems). Then, endogenous peroxidase activity was blocked by applying an ultraviolet inhibitor for 4 minutes. After washing with the reaction buffer several times at room temperature (RT), an Ultra View Universal DAB Detection Kit (Ventana Medical Systems) was used for immunohistochemical (IHC) staining. The slides were incubated with monoclonal antibodies against survivin (Abcam, Cambridge, MA, USA) for 1 hour and against CD133/1 (MACS, Miltenyi Biotec, Bergisch Gladbach, Germany) for 2 hours at $37^{\circ} \mathrm{C}$ in an autostainer (Benchmark XT, Ventana Medical Systems). After incubating with the primary antibodies, the slides were rinsed with the reaction buffer. Drops of HRP UNIV MULT, DAB, and DAB $\mathrm{H}_{2} \mathrm{O}_{2}$ (Ventana Medical Systems) were applied sequentially on each slide (8 minutes per reagent), with intermittent rinsing with reaction buffer. The slides were then treated with a drop of COPPER for 4 minutes. Finally, a bluing agent was added and rinsed with reaction buffer. In addition, the sections were counterstained with hematoxylin for nuclei staining.

CD133 and survivin expression was manually counted in selected hot-spot fields.

IHC expression for CD133 was scored as 0 when there was no expression, $1+$ when the expression for $\mathrm{CD} 133$ was detected in $1 \%-10 \%$ of the whole tumor area, and $2+$ and $3+$ when the expression was in $11 \%-50 \%$ and $51 \%-100 \%$ of the tumor area, respectively. We set a cut-off point at $10 \%$ between the positive and negative groups. Survivin expression was scored as $1+(0 \%-25 \%), 2+(26 \%-50 \%), 3+(51 \%-75 \%)$, and $4+(76 \%-$ $100 \%)$ according to the level of nuclear reactivity among total tumor cells. ${ }^{18}$ We set a cut-off point at $25 \%$ between the positive and negative groups. The IHC staining results were independently evaluated by two pathologists who were unaware of patient clinical and pathological information. Discrepancies between the pathologists were resolved by consensus.

\section{Terminal deoxynucleotidyl transferase-mediated deoxyuridine} triphosphate nick-end labeling assay

All patient samples were stained using an In situ Cell Death Detection Kit (Roche Diagnostics, Mannheim, Germany) in formalin-fixed and paraffin-embedded sections. The slides were deparaffinized and rehydrated. After washing, the slides were incubated for 30 minutes at RT with $0.1 \mathrm{M}$ Tris- $\mathrm{HCl}(\mathrm{pH} 7.5)$, $3 \%$ bovine serum albumin, and $20 \%$ normal bovine serum for blocking. Then, the slides were washed twice with phosphatebuffered saline (PBS). The terminal deoxynucleotidyl transferasemediated deoxyuridine triphosphate nick-end labeling (TUNEL) reaction mixture $(50 \mu \mathrm{L})$ was applied to each section for 1 hour at $37^{\circ} \mathrm{C}$ in a humidified chamber. After three washes with PBS, the slides were treated with $0.3 \% \mathrm{H}_{2} \mathrm{O}_{2}$ in methanol for 10 minutes (RT). The blocking step was then repeated.

After washing with PBS, $50 \mathrm{~mL}$ Converter-POD was applied to each slide, and the slides were incubated for 30 minutes at $37^{\circ} \mathrm{C}$ in a humidified chamber. After three washes with PBS, $100 \mu \mathrm{L}$ DAB substrate solution was added, and the slides were incubated for 1-3 minutes at RT. The slides were then washed thoroughly with tap water and counterstained with hematoxylin before mounting. Under light microscopy, we calculated the apoptotic index (AI) by counting the number of apoptotic nuclei per 1,000 cells in a high-power field. Cells were considered positive if the entire nuclear area of the cell was stained brown. Cells in areas with necrosis and the margins of sections were excluded from the analysis. 


\section{Statistical analysis}

Data are displayed according to the properties of the variable. Continuous variables are presented as mean and standard deviation. Categorical variables are noted as frequency and percentage. The Mantel-Haenszel test was performed to evaluate the linear association between two variables on an ordinal scale. To compare the survivin IHC groups, we performed one-way analysis of variance or the chi-square test (Fisher exact test), as appropriate. The survival curve was estimated by the Kaplan-Meier method and was compared by the log-rank test. To estimate hazard ratio and $95 \%$ confidence interval, we used a Cox proportional hazard regression model. A p-value less than 0.05 was considered statistically significant, and all statistical analyses were performed with SAS ver. 9.2 (SAS Inc., Cary, NC, USA).

\section{RESULTS}

Relationships between survivin expression, clinicopathological parameters, and CD133 expression

The demography of cases examined in this study is presented in Table 1. Representative cases with high and low survivin expression are shown in Fig. 1A-D with matched CD133 expression. CD133 showed a luminal membranous expression pattern (Fig. 1A), whereas survivin showed distinct nuclear expression with weak and vague cytoplasmic expression in CRC cells (Fig. 1B). We analyzed only the nuclear survivin expression in this

Table 1. Patients demographics for the current study

\begin{tabular}{lc}
\hline & No. (\%) \\
\hline Sex & $117(62.5)$ \\
Male & $70(37.4)$ \\
Female & \\
Age (yr) & $156(83.4)$ \\
$\geq 50$ & $31(16.6)$ \\
$<50$ & \\
Tumor differentiation grade & $10(5.3)$ \\
Well differentiated & $159(85)$ \\
Moderately differentiated & $18(9.6)$ \\
Poorly differentiated & $72(38.5)$ \\
Tumor stage & $115(61.5)$ \\
II & \\
III & $44(23.5)$ \\
Location & $64(34.2)$ \\
Cecum, appendix, and ascending colon & $79(42.2)$ \\
Transverse, descending, and sigmoid colon & $73(39)$ \\
Rectum & $70(37.4)$ \\
Regional lymph node & $44(23.5)$ \\
N0 & \\
N1 & \\
\hline N2 & \\
\hline
\end{tabular}
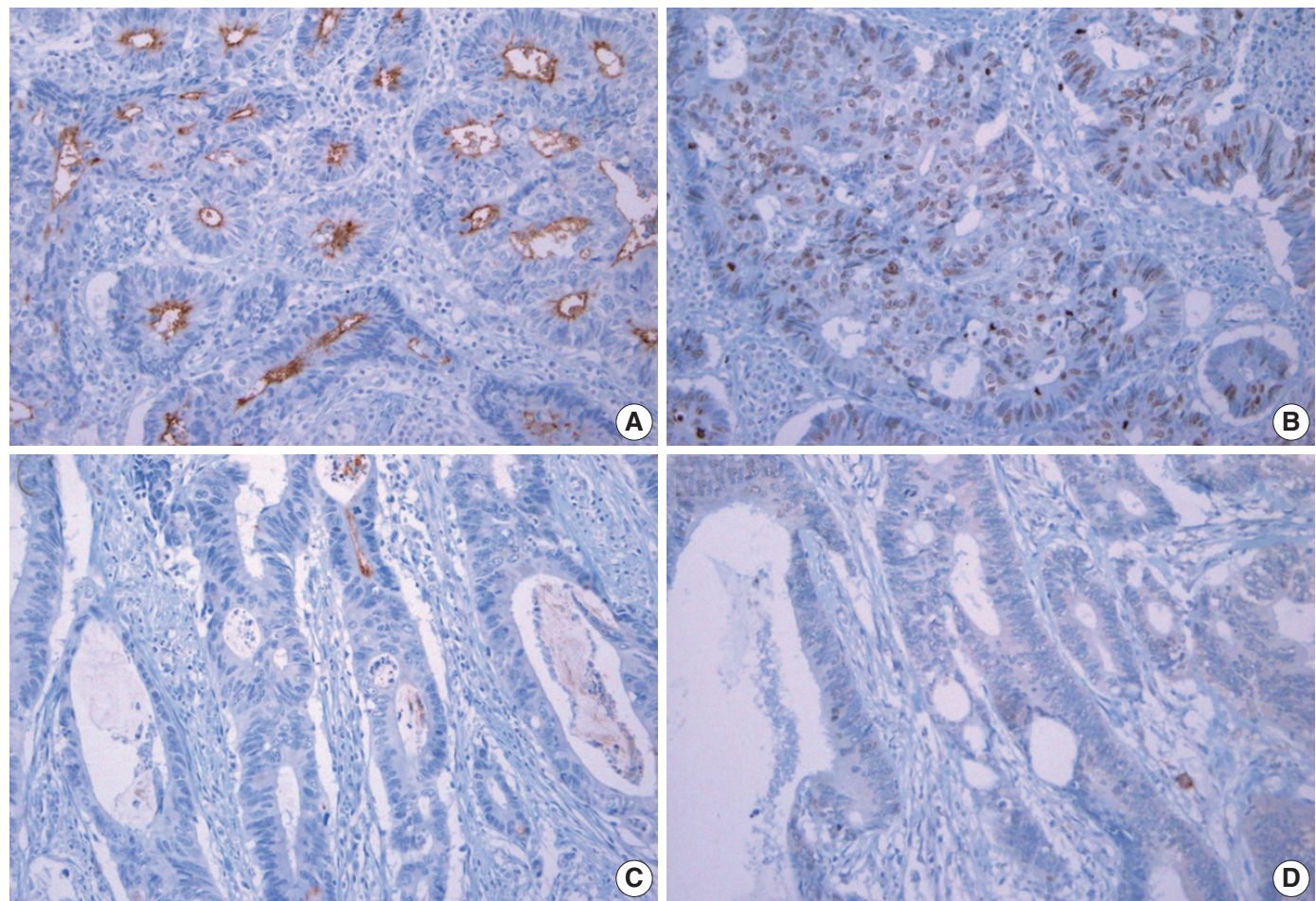

Fig. 1. Photomicrographs showing representative cases of high CD133 (A) and survivin (B) expression and low CD133 (C) and survivin (D) expression in colorectal cancer tumor cells. CD133 expression localized to the luminal surface of the cytoplasm, and survivin expression localized to the nucleus. 
study because the cytoplasmic expression was too diffuse and weak to be scored.

Survivin expression showed a significant correlation with CD133 expression ( $\mathrm{p}=.018$ ) when analyzed by the MantelHaenszel test; however, survivin showed no significant relationship with other pathological parameters (Table 2).

\section{Survival analysis according to survivin expression}

The mean overall survival (OS) according to survivin expression was $113.1 \pm 9.1$ months for score $1+, 47.0 \pm 1.7$ months for score $2+, 41.8 \pm 1.4$ months for score $3+$, and $7.4 \pm 0.1$ months for score $4+$. However, survivin expression had no prognostic significance in the survival analysis $(p=.480)$. Disease-free survival was also not related to survivin expression ( $\mathrm{p}$ $=.488)$.

\section{Difference in apoptosis according to survivin and CD133 expression}

Apoptosis detected by TUNEL analysis showed rare positive cells in normal mucosa (Fig. 2A), in contrast to occasionally scattered positive cells in CRC cells (Fig. 2B). AI was significantly higher in $\mathrm{CD} 133^{+}$tumors $(5.165 \pm 4.961)$ than in $\mathrm{CD} 133^{-}$

Table 2. Survivin expression according to the pathologic parameters $(n=187)$

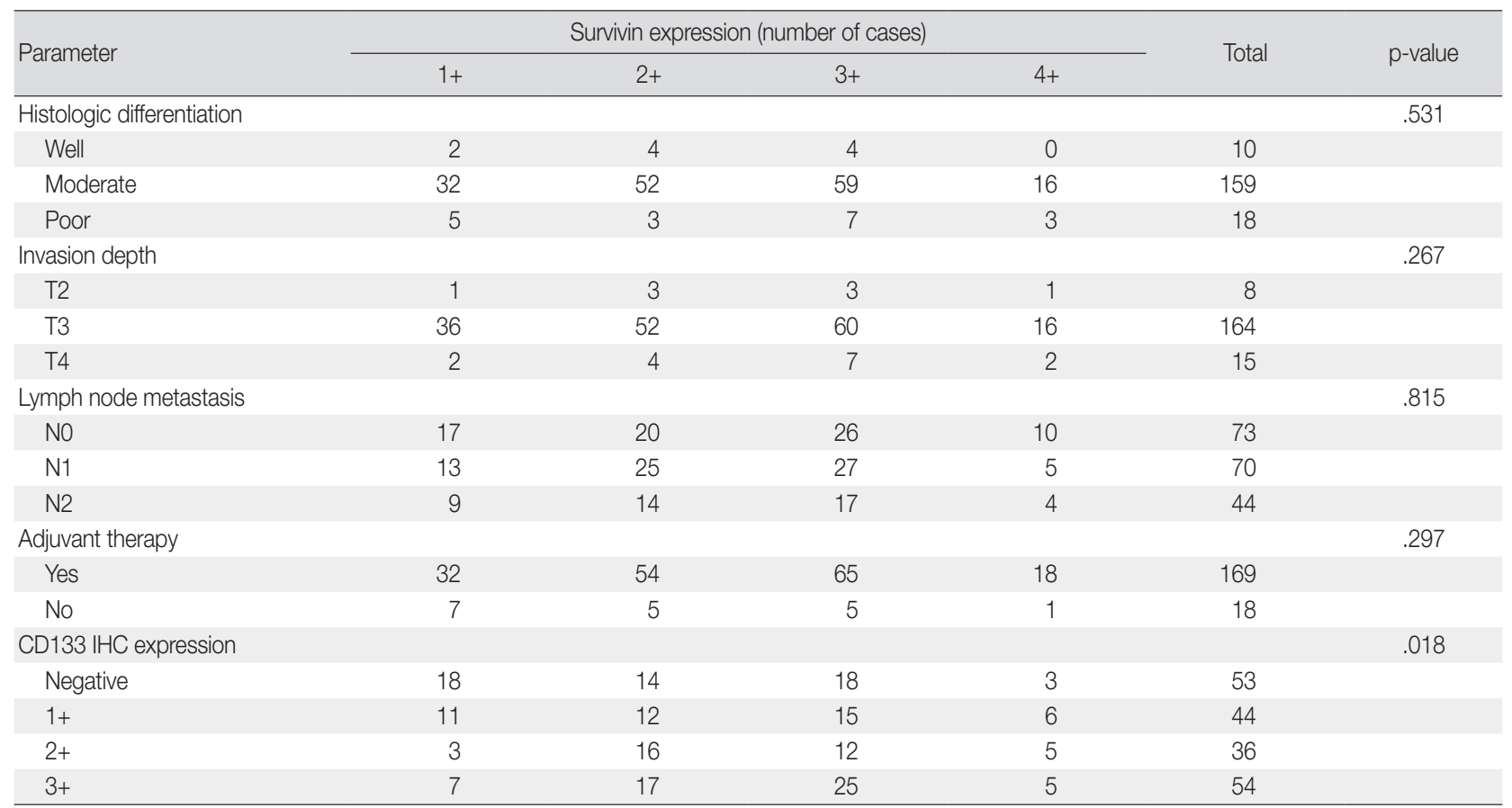

IHC, immunohistochemistry.
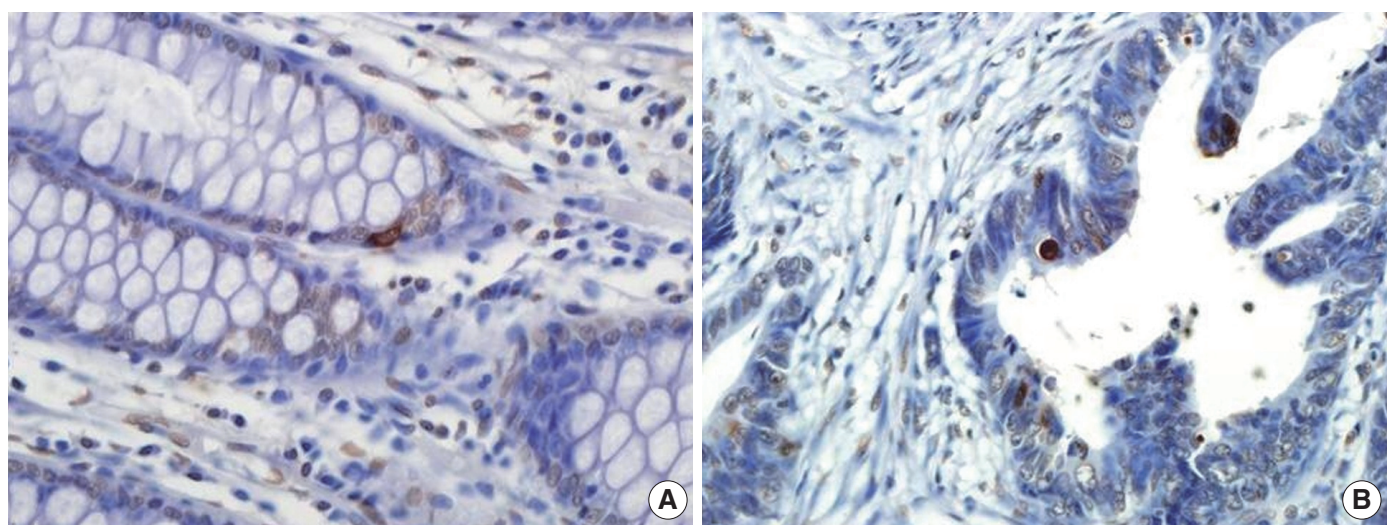

Fig. 2. There was rare apoptosis in normal mucosa (A), but scattered positively-stained apoptotic cells in colorectal cancer (B) were observed by the terminal deoxynucleotidyl transferase-mediated deoxyuridine triphosphate nick-end labeling assay. 


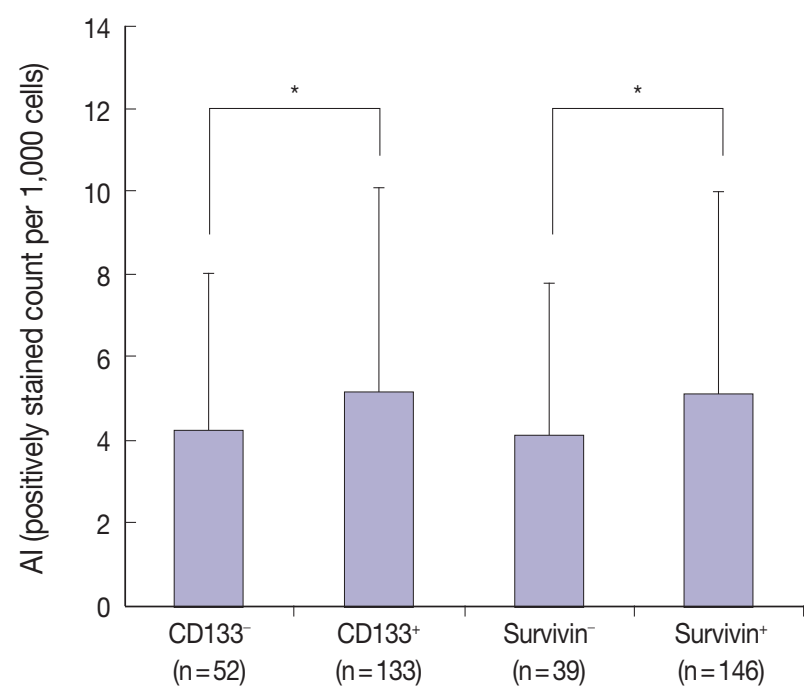

Fig. 3. Compared with that in CD133 and survivin tumors, the mean apoptotic index (Al) obtained by the transferase-mediated deoxyuridine triphosphate nick-end labeling assay was significantly higher in $\mathrm{CD}_{133^{+}}$and survivin ${ }^{+}$tumors $\left({ }^{*} \mathrm{p}<.05\right)$.

tumors $(4.231 \pm 3.812, \mathrm{p}=.034)$. Further, compared with survivin $^{-}$tumors, survivin ${ }^{+}$tumors had a higher $\mathrm{AI}(5.116 \pm 4.894$ vs $4.103 \pm 3.691, \mathrm{p}=.044)$ (Fig. 3).

\section{DISCUSSION}

In our previous in vitro study, we described that $\mathrm{CD} 133^{+}$colon cancer cells showed chemoresistance to 5-FU through high survivin expression. In addition, we demonstrated that the survivin inhibitor YM155 was more effective than 5-FU in inducing cell death of colon cancer cells. ${ }^{4}$ YM155 is a novel small-molecule survivin inhibitor that acts by inhibiting survivin promoter activity. ${ }^{19}$ Inhibitors of apoptosis have been used as new targeted therapies in CRC. ${ }^{20}$ Recently, Gyuraszova et al. ${ }^{21}$ also reported that the combined use of YM155 with hypericin is effective in the treatment of CRC and lung adenocarcinoma through induction of apoptosis. To determine whether the results of our in vitro study are applicable to clinical samples, we examined the significance of survivin expression in relation to clinicopathological parameters and CD133 expression of CRC in the present study. We found a correlation between CD133 expression and nuclear survivin expression in CRC in a sample size of 187 . It agrees with our previous in vitro study, which indicates that both the mRNA and protein expression levels of survivin in $\mathrm{CD} 133^{+}$cells are remarkably higher than those in small interfering RNA (siRNA)induced $\mathrm{CD} 133^{-}$cells, ${ }^{3}$ These findings are also concordant with those of the transcriptome analysis reported by Kim et al. ${ }^{22}$
In two recently published meta-analyses, the authors concluded that survivin expression is closely associated with high mortality. ${ }^{16,23}$ However, these studies did not specify whether the positive correlation was observed with nuclear or cytoplasmic survivin expression. In most data used in these meta-analyses, the subcellular localization of survivin expression was unstated or cytoplasmic. As previously mentioned, nuclear survivin expression can improve cell proliferation by regulating chromosome alignment and segregation in mitosis, whereas cytoplasmic survivin expression inhibits the apoptosis pathway. Another recent report suggested that nuclear survivin expression is related to better patient prognosis. ${ }^{17}$ However, this finding is contrasted with results reported by Kim et al., ${ }^{22}$ who showed that nuclear survivin expression can be an independent poor prognostic factor for disease-free survival and OS. It remains controversial whether nuclear survivin expression is related to patient prognosis. Nuclear survivin expression has been reported to be significantly higher than cytoplasmic expression in CRC. ${ }^{24}$ Therefore, further largescale studies are required to confirm the prognostic significance of subcellular survivin expression in CRC.

In a previous study, we found that $\mathrm{CD} 133$ expression was related to survivin expression in CRC cell lines. ${ }^{3,4}$ However, we found that survivin expression increased in Caco-2 cells after transfection with Ctrl-siRNA and CD133-siRNA. This may indicate that transfection affects survivin expression as a cell-damaging force. To avoid the bias induced by transfection, we analyzed survivin and CD133 expression together with apoptosis in clinical samples of CRC.

The balance between proliferation and apoptosis maintains the homeostasis of the colonic epithelium, but uncontrolled cell proliferation and apoptosis contribute to tumor development. ${ }^{18}$ Alcaide et al. ${ }^{13}$ reported that $\mathrm{AI}$ in $\mathrm{CRC}$ is higher than that in adenomas and normal tissue. We also found that $\mathrm{AI}$ in tumor tissue was higher than that in the surrounding normal mucosa. In addition, $\mathrm{CD} 133^{+}$and survivin ${ }^{+}$tumors showed a higher mean $\mathrm{AI}$ than $\mathrm{CD} 133^{\circ}$ and survivin tumors. However, AI was not significantly related with either survivin or CD133 expression in this study. Bedi et al ${ }^{25}$ reported that apoptosis is inhibited during CRC development, owing to abnormal expression of the BCL2 gene. Zhao et al. ${ }^{26}$ reported that a low dose of bcl-2 inhibitor could up-regulate survivin expression in hepatocellular carcinoma. These results indicate that apoptosis is regulated by not only survivin, but also bcl-2. In addition, the effects of apoptotic proteins on $\mathrm{CD}_{13} 3^{+}$colon cancer cells have been rarely reported. In a study by Kemper et al. ${ }^{27}$ activated caspase- 9 was found to induce a high level of apoptosis in $\mathrm{CD} 133^{+} \mathrm{CRC}$ stem cells. 
Sam et al. ${ }^{28}$ demonstrated that inhibiting survivin and caspase 3 triggers apoptosis in colon cancer stem-like cells.

In this study, we found that nuclear survivin expression was correlated with CD133 expression in CRC, although it was not significantly related to pathological parameters or patient prognosis. Further in vivo investigations of the effect of survivin inhibitors and the mechanism of survivin expression in relation to cancer stem cell markers are required for verifying whether survivin is a new target for more effective treatment of CRC.

\section{Conflicts of Interest}

No potential conflict of interest relevant to this article was reported.

\section{Acknowledgments}

This study was supported by a research grant from the Korea Science and Engineering Foundation funded by the Korean government (Ministry of Education, Science, and Technology, grant code: 2015-51-0329).

\section{REFERENCES}

1. Ferlay J, Soerjomataram I, Dikshit R, et al. Cancer incidence and mortality worldwide: sources, methods and major patterns in GLOBOCAN 2012. Int J Cancer 2015; 136: E359-86.

2. Ren F, Sheng WQ, Du X. CD133: a cancer stem cells marker, is used in colorectal cancers. World J Gastroenterol 2013; 19: 2603-11.

3. Lee MR, Ji SY, Mia-Jan K, Cho MY. Chemoresistance of CD133(+) colon cancer may be related with increased survivin expression. Biochem Biophys Res Commun 2015; 463: 229-34.

4. Li WL, Lee MR, Cho MY. The small molecule survivin inhibitor YM155 may be an effective treatment modality for colon cancer through increasing apoptosis. Biochem Biophys Res Commun 2016; 471: 309-14.

5. Sung JJ, Noh SJ, Bae JS, et al. Immunohistochemical expression and clinical significance of suggested stem cell markers in hepatocellular carcinoma. J Pathol Transl Med 2016; 50: 52-7.

6. Dalerba P, Dylla SJ, Park IK, et al. Phenotypic characterization of human colorectal cancer stem cells. Proc Natl Acad Sci U S A 2007; 104: 10158-63.

7. Deng YH, Pu XX, Huang MJ, et al. 5-Fluorouracil upregulates the activity of Wnt signaling pathway in CD133-positive colon cancer stem-like cells. Chin J Cancer 2010; 29: 810-5.

8. Corbo C, Orrù S, Gemei M, et al. Protein cross-talk in CD133+ colon cancer cells indicates activation of the Wnt pathway and up- regulation of SRp20 that is potentially involved in tumorigenicity. Proteomics 2012; 12: 2045-59.

9. Sikandar SS, Pate KT, Anderson S, et al. NOTCH signaling is required for formation and self-renewal of tumor-initiating cells and for repression of secretory cell differentiation in colon cancer. Cancer Res 2010; 70: 1469-78.

10. Lin L, Fuchs J, Li C, Olson V, Bekaii-Saab T, Lin J. STAT3 signaling pathway is necessary for cell survival and tumorsphere forming capacity in $\mathrm{ALDH}(+) / \mathrm{CD} 133(+)$ stem cell-like human colon cancer cells. Biochem Biophys Res Commun 2011; 416: 246-51.

11. Roy S, Majumdar AP. Signaling in colon cancer stem cells. J Mol Signal 2012; 7: 11.

12. Dick JE. Looking ahead in cancer stem cell research. Nat Biotechnol 2009; 27: 44-6.

13. Alcaide J, Funez R, Rueda A, et al. The role and prognostic value of apoptosis in colorectal carcinoma. BMC Clin Pathol 2013; 13: 24.

14. Mobahat M, Narendran A, Riabowol K. Survivin as a preferential target for cancer therapy. Int J Mol Sci 2014; 15: 2494-516.

15. Huang YJ, Qi WX, He AN, Sun YJ, Shen Z, Yao Y. The prognostic value of survivin expression in patients with colorectal carcinoma: a meta-analysis. Jpn J Clin Oncol 2013; 43: 988-95.

16. Krieg A, Werner TA, Verde PE, Stoecklein NH, Knoefel WT. Prognostic and clinicopathological significance of survivin in colorectal cancer: a meta-analysis. PLoS One 2013; 8: e65338.

17. Qi G, Tuncel H, Aoki E, et al. Intracellular localization of survivin determines biological behavior in colorectal cancer. Oncol Rep 2009; 22: 557-62.

18. De Oliveira Lima F, De Oliveira Costa H, Barrezueta LF, et al. Immunoexpression of inhibitors of apoptosis proteins and their antagonist SMAC/DIABLO in colorectal carcinoma: correlation with apoptotic index, cellular proliferation and prognosis. Oncol Rep 2009; 22: 295-303.

19. Rauch A, Hennig D, Schäfer C, et al. Survivin and YM155: how faithful is the liaison? Biochim Biophys Acta 2014; 1845: 202-20.

20. Miura K, Fujibuchi W, Ishida K, et al. Inhibitor of apoptosis protein family as diagnostic markers and therapeutic targets of colorectal cancer. Surg Today 2011; 41: 175-82.

21. Gyurászová K, Mikeš J, Halaburková A, Jendželovský R, Fedoročko P. YM155, a small molecule inhibitor of survivin expression, sensitizes cancer cells to hypericin-mediated photodynamic therapy. Photochem Photobiol Sci 2016; 15: 812-21.

22. Kim ST, Sohn I, Do IG, et al. Transcriptome analysis of CD133-positive stem cells and prognostic value of survivin in colorectal cancer. Cancer Genomics Proteomics 2014; 11: 259-66.

23. Li Y, Ma X, Wu X, Liu X, Liu L. Prognostic significance of survivin in breast cancer: meta-analysis. Breast J 2014; 20: 514-24. 
24. Shintani M, Sangawa A, Yamao N, Kamoshida S. Immunohistochemical expression of nuclear and cytoplasmic survivin in gastrointestinal carcinoma. Int J Clin Exp Pathol 2013; 6: 2919-27.

25. Bedi A, Pasricha PJ, Akhtar AJ, et al. Inhibition of apoptosis during development of colorectal cancer. Cancer Res 1995; 55: 1811-6.

26. Zhao X, Ogunwobi OO, Liu C. Survivin inhibition is critical for $\mathrm{Bcl}-2$ inhibitor-induced apoptosis in hepatocellular carcinoma cells. PLoS One 2011; 6: e21980.
27. Kemper K, Rodermond H, Colak S, Grandela C, Medema JP. Targeting colorectal cancer stem cells with inducible caspase-9. Apoptosis 2012; 17: 528-37.

28. Sam S, Sam MR, Esmaeillou M, Safaralizadeh R. Effective targeting survivin, caspase-3 and microRNA-16-1 expression by methyl3-pentyl-6-methoxyprodigiosene triggers apoptosis in colorectal cancer stem-like cells. Pathol Oncol Res 2016; 22: 715-23. 